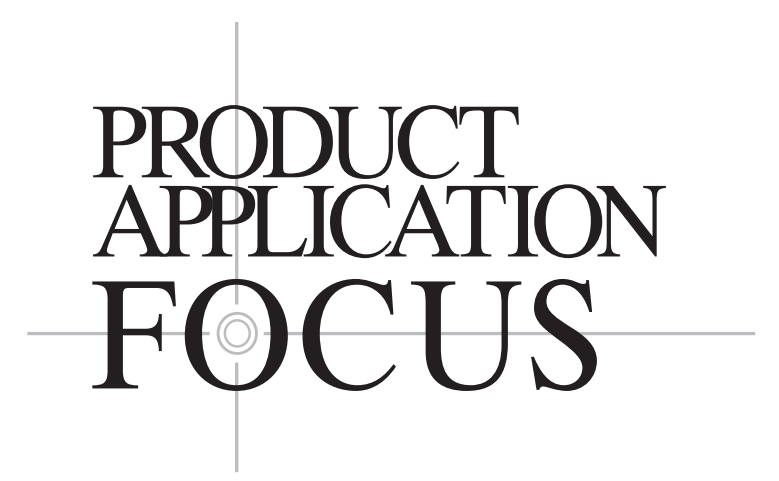

\title{
DNA Sequencing Using Rolling Circle Amplification and Precision Glass Syringes in a High-Throughput Liquid Handling System
}

\author{
Hui-Chung Wu, Jean Shieh, David J. Wright, and Arezou Azarani \\ Apogent Discoveries, Sunnyvale, CA, USA
}

BioTechniques 34:204-207 (January 2003)

\begin{abstract}
An automated high-throughput method that employs rolling circle amplification (RCA) to generate template for large-scale DNA sequencing has been developed using liquid handling systems equipped with precision glass syringes. A protocol was designed to perform the sequencing analysis from template preparation to thermal cycle sequencing within the same vessel, thus minimizing the amount of liquid handling and transfer. The amplified DNA was directly used for cycle sequencing with no need for any purification procedures. Total $R C A$ reaction volumes as low as $500 \mathrm{~nL}$ generated sufficient templates for successful sequencing. Reducing the RCA total reaction volumes by a 40-fold factor, from a total of $20 \mu \mathrm{L}$ to 500 $n L$, resulted in a significant reduction in cost, from $\$ 1.25 /$ reaction to less than \$0.04/reaction. Additionally, the volume of the sequencing reactions was reduced from a total of 20 to $10 \mu \mathrm{L}$, thus generating a further cost advantage. This high-throughput DNA sequencing protocol maximizes the speed and precision of processing while significantly reducing the cost of amplification.
\end{abstract}

\section{INTRODUCTION}

A major processing bottleneck in large-scale sequencing projects is the amplification and purification of DNA templates. Two popular methods for amplifying templates are PCR (4) and the propagation of plasmids in biological hosts followed by their isolation (plasmid purification) (5). Both procedures require many complex and laborious steps that are difficult to automate and control. For example, plasmid am- plification requires a bacterial growth step followed by lysis and purification. This method takes $24 \mathrm{~h}$ to complete, and the DNA produced varies in both yield and quality. PCR amplification is much faster; however, it is very susceptible to contamination and mis-priming, which makes it unreliable for large-scale work. Furthermore, the PCR-amplified products must be purified and may contain sequence errors, which could reduce sequencing quality.

Recently a DNA amplification kit was developed based on the replication of circular DNA molecules in nature. The TempliPhi $^{\text {TM }}$ DNA Sequencing Template Amplification Kit from Amersham Biosciences (Piscataway, NJ, USA) employs bacteriophage Phi29 DNA polymerase to generate template by rolling circle amplification (RCA) (2,3). Phi29 DNA polymerase is a highly processive proofreading, robust enzyme with high strand displacement activity on replication forks. Using random hexamer primers to initiate synthesis, a large quantity of tandem copies can be created from the starting circular DNA (amplification level of $10^{9}$-fold). RCA is less prone to contamination and errors than PCR-based amplification. This advantage of RCA over PCR-based amplification is because, in PCR amplification, the newly synthesized molecules serve as templates for further synthesis, while in RCA, the original template is copied numerous times (1). RCA (an isothermal method performed at $30^{\circ} \mathrm{C}$ ) generates sufficient high-quality templates, which can be used for cycle sequencing without any purification requirements. Therefore, RCA is a simplified procedure that can replace the conventional methods of template preparation for high-throughput sequencing.

The simplicity of the RCA method makes it an excellent 
means for automation and miniaturization. Miniaturization of reaction volumes, without sacrificing the quality of result or increasing the performance cost, increases throughput. In this study, an automated high-throughput method that employs RCA to generate template for large-scale DNA sequencing has been developed using the Robbins ${ }^{\circledR}$ Tango ${ }^{\mathrm{TM}}$ and Hydra $^{\circledR}{ }^{\circledR}$-PP liquid handling systems (Apogent Discoveries, Sunnyvale, CA, USA) equipped with 1-384 precision glass syringes for all dispensing purposes. Precision glass syringes offer a reliable and accurate method of performing low-volume liquid dispensing. They can be used to dispense volumes as low as $100 \mathrm{~nL}$ with very high precision (in the order of $90 \%$ ). In addition to automation, these nanoliter dispensing instruments were used for miniaturizing the amplification and sequencing procedures (RCA reaction volume was reduced by a factor of 40 , and sequencing reaction volume was reduced by a factor of 2), therefore achieving significant cost savings. To minimize the amount of liquid handling and transfer, thus maximizing the speed of processing, a novel protocol was designed to perform the sequencing analysis from template preparation to thermal cycle sequencing within the same vessel. This unique high-throughput DNA sequencing protocol applies the low-volume dispensing capabilities of precision glass syringes in automated systems to maximize the speed and precision of processing while significantly reducing the cost of amplification.

\section{MATERIALS AND METHODS}

TempliPhi DNA Sequencing Template Amplification Kit and bacterial cultures containing plasmid pUC 18 were provided by the U.S. Department of Energy's Joint Genome In- stitute (Walnut Creek, CA, USA). A Tango Liquid Handling System and a Hydra Microdispenser equipped with 384 100$\mu \mathrm{L}$ stainless-steel syringes with Teflon ${ }^{\circledR}$ coating were used for liquid handling purposes. Agarose gel electrophoresis was performed as the standard protocol to check the quality/quantity of the RCA products. $1 \mathrm{~Kb}$ Plus DNA Ladder was purchased from Invitrogen (Carlsbad, CA, USA). Electrophoresis images were detected with a NucleoVision ${ }^{\mathrm{TM}}$ Imaging Workstation from NucleoTech (San Mateo, CA, USA). The GeneQuant II RNA/DNA Calculator from Amersham Biosciences (Cambridge, UK) was used for DNA quantification. DNA sequencing reactions were performed using $\mathrm{ABI}$ PRISM $^{\circledR}$ BigDye ${ }^{\mathrm{TM}}$ Terminator Cycle Sequencing Ready Reaction Kit from Applied Biosystems (Foster City, CA, USA).

\section{Preparation of the RCA Reagents and the Liquid Handling Systems}

Before the setup of the RCA reactions, both the Hydra and Tango systems' dispensing syringes were washed with the following specific protocols. The Hydra syringes were washed three times with distilled water, with each wash composed of three wash cycles ("one wash cycle" was defined as a fill and empty at full syringe volume). The Tango syringes were washed with one three-cycle wash with distilled water. Next, the syringes on both systems were washed with $70 \%$ ethanol for three cycles and then dried overnight. Before their use for dispensing RCA reagents, the syringes were once again washed with distilled water for three cycles and then rinsed three times with $5 \mathrm{mg} / \mathrm{mL}$ BSA to coat the needle surfaces. The BSA coating prevents DNA polymerase activity loss. BSA was also added to the TempliPhi Kit's enzyme premixture to a final concentration of $25 \mathrm{ng} / \mu \mathrm{L}$. Before the start of the amplification experiments, a master mixture was prepared by adding $2 \mu \mathrm{L}$ bacterial sample to $10 \mu \mathrm{L}$ TempliPhi Kit denaturing buffer. This mixture was heated to $95^{\circ} \mathrm{C}$ for $3 \mathrm{~min}$ and cooled before the setup of the RCA reactions.

Since nondisposable precision glass syringes are used for all dispensing, it is crucial to follow an efficient syringecleaning procedure for inhibition of sample-to-sample carryover contamination between dispenses (Robbins Application Notes 5 and 10; http://www. robsci.com/hug.html) (6).

Automated Dispensing Precision of the Liquid Handling Systems

Before setting up the RCA reactions, the uniformity and consistency of the volumes dispensed across the array of 384 syringes in the Hydra and Tango systems were determined by the $\mathrm{CV}$ for specific dispensing 
volumes (6). A high precision in dispensing volumes as low as $100 \mathrm{~nL}$ with the Tango and the Hydra systems was evidenced by cVs of less than $10 \%$.

\section{Dispensing the RCA Reagents with Precision Glass Syringes in Automated Liquid Handling Systems}

Using a Hydra microdispenser equipped with $384100-\mu \mathrm{L}$ precision glass syringes with stainless-steel needles, $2 \mu \mathrm{L}$ mineral oil were dry dispensed into a 384 CyclePlate $^{\circledR}$ (Apogent Discoveries). Using a second Hydra system, $500 \mathrm{~nL}$ enzyme/BSA premixture and then $500 \mathrm{~nL}$ of the bacteria/ buffer mixture were dispensed under the mineral oil. The RCA procedure was repeated as described above but using a Tango liquid handling system equipped with $384100-\mu \mathrm{L}$ syringes with DuraFlex ${ }^{\mathrm{TM}}$ needles. To reduce the total RCA reaction volumes further, $250 \mathrm{~nL}$ enzyme/BSA premixture and $250 \mathrm{~nL}$ bacteria/buffer mixture were dispensed under $2 \mu \mathrm{L}$ mineral oil using the Tango system. The samples were incubated at $30^{\circ} \mathrm{C}$ for $18 \mathrm{~h}$ (or room temperature for a period of 48 h). To deactivate the enzyme, samples were heated at $96^{\circ} \mathrm{C}$ for $5 \mathrm{~min}$ and then stored at $4^{\circ} \mathrm{C}$ until ready to proceed with setting up the sequencing reactions. The quantity and quality

A

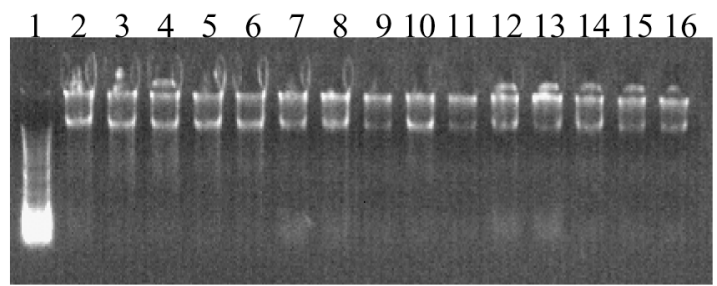

B

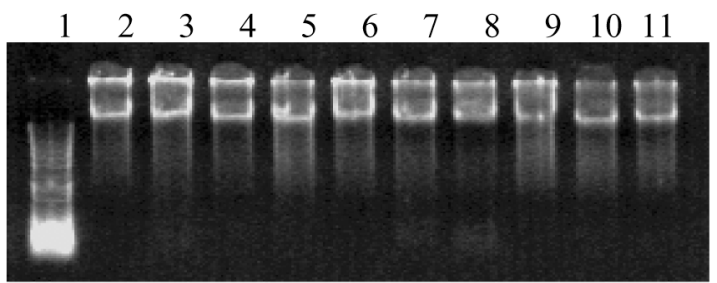

C

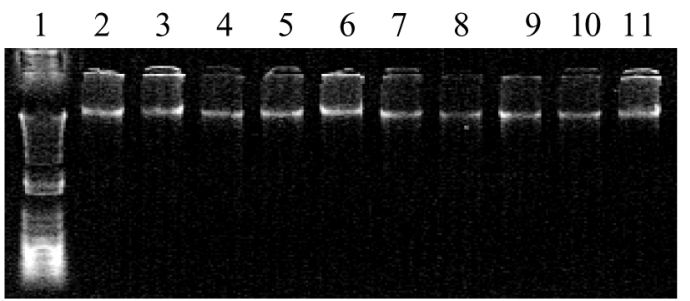

Figure 2. Electrophoresis analysis of the RCA reactions. (A) A $0.6 \%$ agarose gel analysis of $1 \mu \mathrm{L}$ RCA reactions prepared with a Hydra microdispenser equipped with $384100-\mu \mathrm{L}$ stainless-steel syringes with Teflon coating. Lane $1,1 \mathrm{~Kb}$ plus DNA ladder. Lanes $2-16$, random samples from a 384well reaction plate. (B) A $0.6 \%$ agarose gel analysis of $1 \mu \mathrm{L}$ RCA reactions prepared with a Tango liquid handling system equipped with 384 100- $\mu \mathrm{L} \mathrm{Du}-$ raFlex syringes. Lane 1, $1 \mathrm{~Kb}$ Plus DNA ladder. Lanes 2-11, random samples from a 384-well reaction plate. (C) A $0.6 \%$ agarose gel analysis of $0.5 \mu \mathrm{L}$ RCA reactions prepared with a Tango liquid handling system equipped with $384100-\mu$ L DuraFlex syringes. Lane 1, 1 Kb Plus DNA ladder. Lanes 2-11, random samples from a 384-well reaction plate. of the amplified templates were determined by $\mathrm{A}_{260 / 280}$ spectrophotometric readings and gel electrophoresis analysis.

\section{Quality Determination by Sequencing}

For determining the quality of the amplified DNA templates, representative DNA samples were sequenced directly (without purification) on an ABI 3700 DNA Analyzer. Template preparation and thermal cycle sequencing were performed in the same 384-well plate. The total sequencing reaction volume was reduced 2-fold from 20 to $10 \mu \mathrm{L}$. In short, 4 $\mu \mathrm{L}$ BigDye Terminator Ready Reaction Mix, $2 \mu \mathrm{L}$ M13 Forward Primer $(5 \mathrm{ng} / \mu \mathrm{L}$ ), and water (to bring the total reaction volume to $10 \mu \mathrm{L}$ ) were added below the mineral oil to the amplified samples ( 1 or $0.5 \mu \mathrm{L}$ ) with a Hydra or a Tango system used for all dispensing. The plates were sealed and placed in an MJR DNA engine for thermal cycle sequencing. The samples were sequenced on an ABI 3700 Analyzer.

\section{RESULTS AND DISCUSSION}

\section{Determining the Quantity and Quality of the Rolling Circle-Amplified DNA}

The TempliPhi Kit recommends setting up a total of $20 \mu \mathrm{L}$ reaction volume for the amplification of micrograms of DNA samples. However, the amount of template required in a cycle sequencing reaction is less than $500 \mathrm{ng}$. Therefore, only aliquots of the $20-\mu \mathrm{L}$ RCA sample are used for cycle sequencing (typically $1-2 \mu \mathrm{L}$ of the RCA product are used in a $20-\mu \mathrm{L}$ cycle sequencing reaction). For this purpose, the RCA reaction volumes were minimized by a factor of 40 (from 20 to $0.5 \mu \mathrm{L}$ ) in this study to reduce the reagent cost per reaction from $\$ 1.25$ to approximately $\$ 0.04$. A protocol that applies the nanoliter dispensing capabilities of precision glass syringes in automated systems to perform template preparation and cycle sequencing at miniaturized volumes was set up (Figure 1). Following the outlined procedure (while using a single Tango instrument attached to a plate stacker), approximately $3072(8 \times 384$-well plates $)$ amplification and sequencing reactions could be prepared per hour.

The automation efficiency of setting up high-throughput RCA to generate template for large-scale DNA sequencing, using liquid handling systems equipped with precision glass syringes, was evaluated by determining the quantity and quality of the amplified samples. The amplified samples were quantified by gel electrophoresis and spectrophotometric analysis. The quantification results indicated that on average a total of $552 \pm 71$ and $209 \pm 60$ ng DNA were amplified in an RCA total reaction volume of 1 and $0.5 \mu \mathrm{L}$, respectively. Reproducibility in the rate of DNA amplification (in a 384-well plate) was evidenced by CVs of less than $15 \%$ for the $1-\mu \mathrm{L}$ total reaction volumes and less than $30 \%$ for the $0.5-\mu \mathrm{L}$ total RCA reaction volumes. The CVs obtained for the quantification results are from three 384 plates for each volume (total of 1152 samples for each volume). Uniformity and consistency in the RCA yields among the wells indicate that automated systems equipped with precision glass syringes efficiently allow for a reproducible method of low-volume dispensing. The superior quality and quantity of the amplified products were also confirmed by gel electrophoresis analysis (Figure 2). 
A

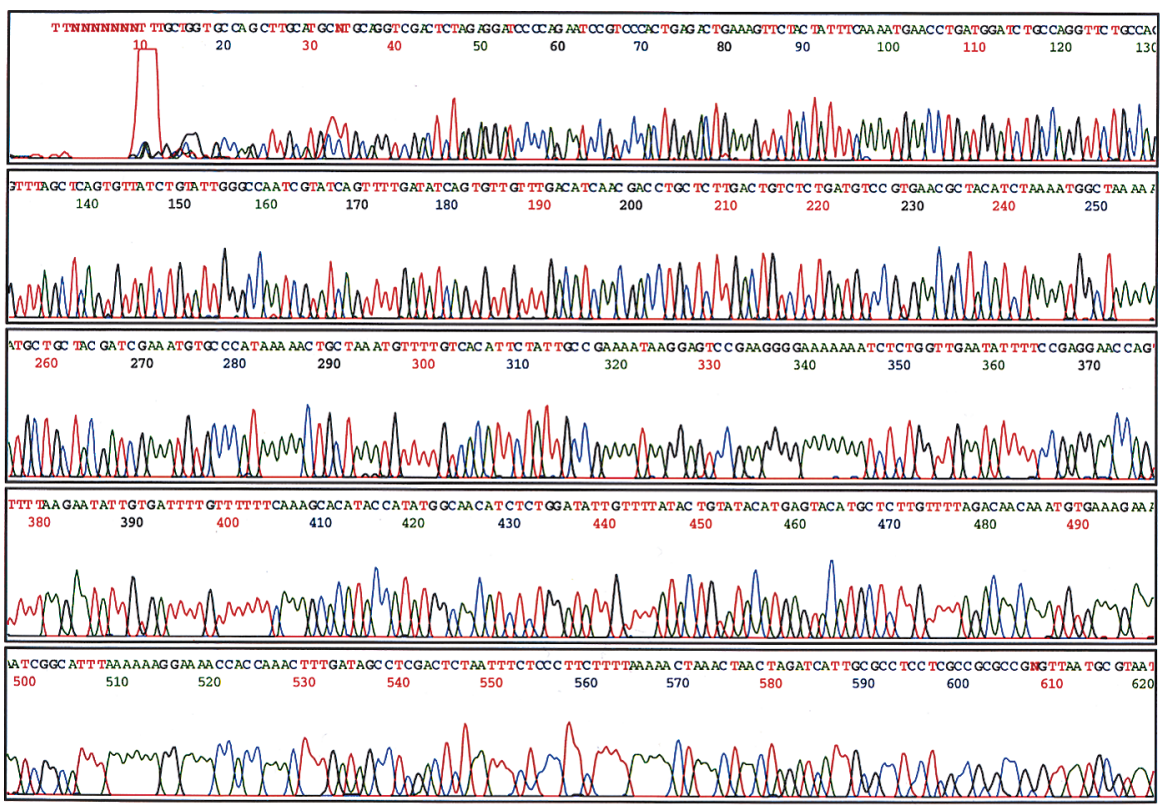

B

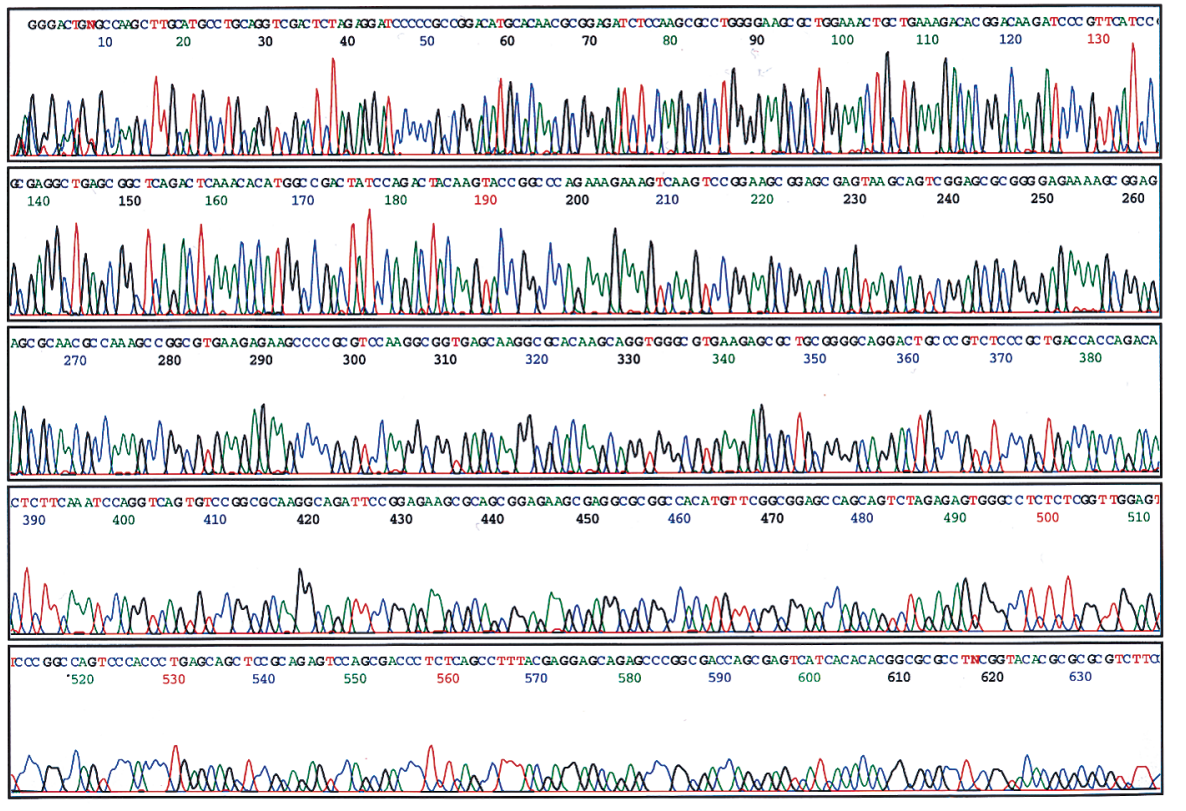

Figure 3. Fluorescent sequence profiles of rolling circle-amplified DNA products. Shown are representative trace data from RCA reaction products sequenced on an ABI 3700 DNA Sequencer. (A) A 1- $\mu$ L total volume RCA reaction product. (B) A $0.5-\mu \mathrm{L}$ total volume RCA reaction product.

\section{Determining the Quality of the Rolling Circle-Amplified DNA by Sequencing}

In addition to reducing the RCA total reaction volumes by a factor of 40 -fold, the volume of the sequencing reactions was reduced by $50 \%$ (from a total of 20 to $10 \mu \mathrm{L}$ ), thus generating a further 2-fold cost advantage. To ascertain the sequencing quality (PHRED Q > 20) of the amplified products, the samples were directly sequenced on an ABI 3700 analyzer. Average PHRED Q $>20$ values of $662 \pm 50$ and $655 \pm 38$ were observed for the 1 - and $0.5-\mu \mathrm{L}$ total RCA reaction volumes, respectively, with a successful rate greater than $95 \%$. A typical ABI BigDye
Terminator cycle sequencing result obtained from the amplified product is shown in Figure 3 for both the 1- and $0.5 \mu \mathrm{L}$ total $\mathrm{RCA}$ reaction volumes.

Miniaturization of the RCA and cycle sequencing reaction volumes had the advantage of generating a significant reduction in the amount of liquid handling and transfer. Since the protocol created in this study was designed to perform the sequencing analysis from template preparation to thermal cycle sequencing within the same vessel, several processing steps were eliminated. As a result, this fully automated protocol maximized the speed and the precision in preparing high-throughput sequencing templates.

Using high-throughput liquid handling systems equipped with precision glass syringes and the RCA technology, a simple, flexible, and precise method of preparing sequencing templates was developed to reduce reagent cost significantly while maximizing the speed and precision of processing.

\section{REFERENCES}

1.Blanco, L., A. Bernad, J.M. Lazaro, G. Martin, C. Garmendia, and M. Salas. 1989. High efficient DNA synthesis by the phage phi 29 DNA polymerase. Symmetrical mode of DNA replication. J. Biol. Chem. 264:8935-8940.

2.Blanco, L., A. Bernad, and M. Salas. 1991. Phi29 DNA Polymerase. United States Patent no. 5001050 .

3.Dean, F.B., J.R. Nelson, T.L. Giesler, and R.S. Lasken. 2001. Rapid amplification of plasmid and phage DNA using Phi29 DNA polymerase and multiply-primed rolling circle amplification. Genome Res. 11:1095-1099.

4.Mullis, K.B., F. Ferre, and R.A. Gibbs. 1994. The Polymerase Chain Reaction. Birkhäuser, Boston, MA.

5.Sambrook, J., E.F. Fritsch, and T. Maniatis. 1989. Molecular Cloning: A Laboratory Manual. CSH Laboratory Press, Cold Spring Harbor, NY.

6.Shieh, J., C. To, J. Carramao, N. Nishimura, Y. Maruta, Y. Hashimoto, D. Wright, H. Wu, and A. Azarani. 2002. High-throughput array production using precision glass syringes. BioTechniques 32:1360-1365.

Address correspondence to Dr. Arezou Azarani, Apogent Discoveries, 1250 Elko Drive, Sunnyvale, CA 94089-2213, USA.e-mail:aazarani@robsci.com 